\title{
Turbine cascade design via multigrid-aided finite-difference progressive optimization
}

\author{
Luciano A. Catalano - Andrea Dadone - Vito S.E. Daloiso \\ Dipartimento di Ingegneria Meccanica e Gestionale, \& Centro di Eccellenza \\ in Meccanica Computazionale,Politecnico di Bari, via Re David 200 Bari, Italy \\ \{catalano,a.dadone,v.daloiso\}@poliba.it
}

ABSTRACT. This paper proposes an efficient and robust procedure for the design optimization of turbomachinery cascades in inviscid and turbulent transonic flow conditions. It employs a progressive strategy, based on the simultaneous convergence of the design process and of all iterative solutions involved (flow analysis, gradient evaluation), also including the global refinement from a coarse to a sufficiently fine mesh. Cheap, flexible and easy-to-program Multigrid-Aided Finite Differences are employed for the computation of the sensitivity derivatives. The entire approach is combined with an upwind finite-volume method for the Euler and the Navier-Stokes equations on cell-vertex unstructured (triangular) grids, and validated versus the inverse design of a turbine cascade. The methodology turns out to be robust and highly efficient, the converged design optimization being obtained in a computational time equal to that required by 15 to 20 (depending on the application) multigrid flow analyses on the finest grid.

RÉSUMÉ. Cet article propose une procédure robuste et efficace pour l'optimisation de formes de turbomachines en écoulements transsoniques, turbulents et non visqueux. On utilise une stratégie progressive, basée sur la convergence simultanée du processus de design et de toutes les solutions itératives considérées (écoulement, gradient), incluant également le raffinement de maillage (de grossier à suffisamment fin). Peu coûteuse, flexible et simple à programmer, une approche différences finies/multigrilles est utilisée pour le calcul des dérivées de sensibilités. L'approche complète est associée à une méthode de volumes finis décentrée pour les équations d'Euler et de Navier-Stokes en maillages non structurés (triangulaires), et validée considérant un problème inverse de cascade de turbine. Cette méthodologie apparaît robuste et efficace, la solution convergée étant obtenue en un temps de calcul équivalent à 15 à 20 (en fonction de l'application considérée) calculs de l'écoulement sur le maillage plus fin.

KEYWORDS: optimization, multigrid, finite difference, turbine cascade.

MOTS-CLÉS : optimisation, multigrille, différences finies, cascade de turbines.

DOI:10.3166/REMN.17.199-215 @ 2008 Lavoisier, Paris

REMN - 17/2008. Shape design in aerodynamics, pages 199 to 215 


\section{Introduction}

In the last years, many CFD researchers have devoted their efforts to the development of robust and efficient gradient-based optimization procedures for the automatic design of fluid-dynamic components. The most ambitious technique developed so far is the so-called one-shot method (Kuruvila et al., 1995; Held et al., 2002), which combines the objective function and the governing equations, so as to define and solve a unique problem. The alternative, iterative formulation of the optimization problem consists of computing the flow on a trial geometry, evaluating the objective function gradient, and, accordingly, modifying the shape. Concerning its efficiency, a progressive optimization strategy has been proposed in (Dadone et al., 2000b), based on the simultaneous convergence of the design process and of all iterative solutions involved (flow analysis, gradient evaluation), also including the global refinement from a coarse to a sufficiently fine mesh. Greater advantage has been taken from the use of multiple grid levels by the methods proposed in (Kuruvila et al., 1995; Mohammadi et al., 2001; Beux et al., 1994), where the descent algorithm and the design variables work according to multigrid (Brandt, 1977) concepts.

Perturbed shapes and finite differences can be used to evaluate the sensitivities. This method is easy-to-program, is invariant with respect to the grid type, to the flow modelization and to the discretization scheme, and can be combined with black-box commercial codes. Its drawback is the large amount of computational work, almost proportional to the number of design parameters. To overcome this low efficiency, adjoint methods, in both continuous and discrete form, have been developed and widely tested, see, e.g., (Jameson, 1988): all sensitivities are computed by solving a unique adjoint system, independently of the number of design parameters. This approach is very efficient, but a very cumbersome, analytical or symbolic, differentiation of the governing equations is required. An alternative, much simpler, approach has been proposed in (Mohammadi, 1997), where it is shown that when the objective function depends on surface integrals, its gradient is not significantly influenced by the flow derivatives, which can be dropped out. The cost of the gradient evaluation becomes negligible, but all applications proposed so far lead to an improvement, rather than to a complete optimization, of the performances of the component under design. An alternative approach, which also takes advantage of the multigrid concepts, but differently from (Kuruvila et al., 1995; Mohammadi et al., 2001; Beux et al., 1994), has been recently proposed by the authors (Catalano et al., 2003a): this Multigrid-Aided FiniteDifference (MAFD) technique should be invariant with respect to the grid type, to the flow modelization and to the discretization scheme, as the standard finite-difference approach. To date, it has been tested, in combination with the progressive optimization strategy of (Dadone et al., 2000b), versus the inviscid transonic flow past a 3D turbine nozzle (Catalano et al., 2003a), using a structured, cell-centered, flow solver and versus the inviscid (transonic and subsonic) and laminar flow past airfoils (Catalano et al., 2005a), using an unstructured, cell-vertex, flow solver. 
This paper proposes the application of the MAFD progressive optimization strategy to the inverse design of turbine cascades in inviscid and turbulent transonic flow conditions, using the aforementioned unstructured, cell-vertex, flow solver.

\section{Flow solver}

An unstructured cell-vertex triangular grid is used to discretize the 2D Euler and Navier-Stokes equations governing the flows considered in this paper.

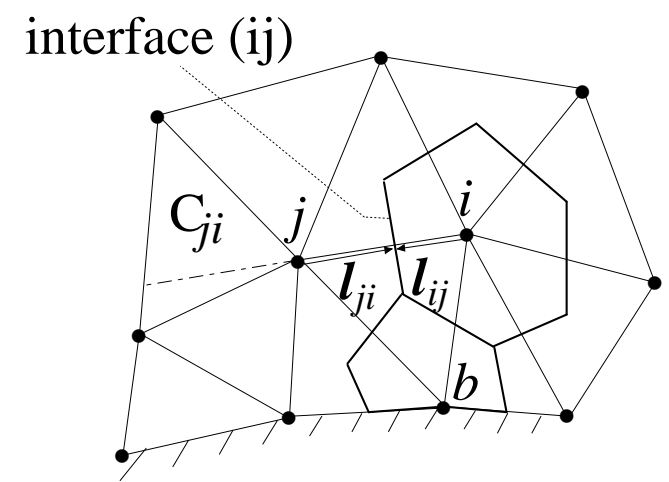

Figure 1. Second-order reconstruction

A left state and a right state are linearly reconstructed on the two sides of each interface $(i j)$, obtained by connecting either the barycenters or the circumcenters of two neighboring triangles. Similarly to the 1-D case, a unique left-neighboring cell is used to define the flow gradient employed in the reconstruction (Catalano, 2002): it is defined as the cell $C_{j i}$ which contains the prolongation of the side $(j i)$, plotted as a dot-dashed line in Figure 1. Standard one-dimensional limiters are also applied straightforwardly. The flux-difference-splitting of (Roe, 1986) is then used to solve the Riemann problem defined at each interface. It is noteworthy that this rather simple method allows to capture discontinuities very sharply, despite the 1-D physics of the Riemann solver (Catalano et al., 2003b). Figure 2 provides the Mach number contours for the flow in a circular-arc bump channel, with $M_{i n l}=1.4$, computed on a grid composed by 15256 cells. Figure 3 shows a zoom of the Mach number contours and of the grid in the $\lambda$-shock region: the shocks are captured in two or three cells; moreover, the $\lambda$-shock is clearly described, and the blending of the downstream Mach number contours reveals the presence of the resulting contact discontinuity.

A standard finite-element Galerkin discretization is used for the viscous terms. Turbulent computations have been performed by means of a standard formulation of the two-equation $k-\omega$ model of Wilcox (Wilcox, 1988). 


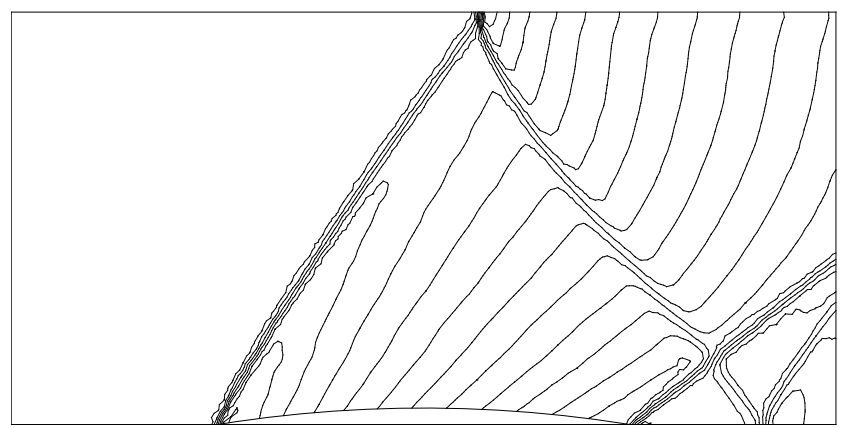

Figure 2. Circular-arc bump channel: Mach number contours $(\Delta M=0.05)$

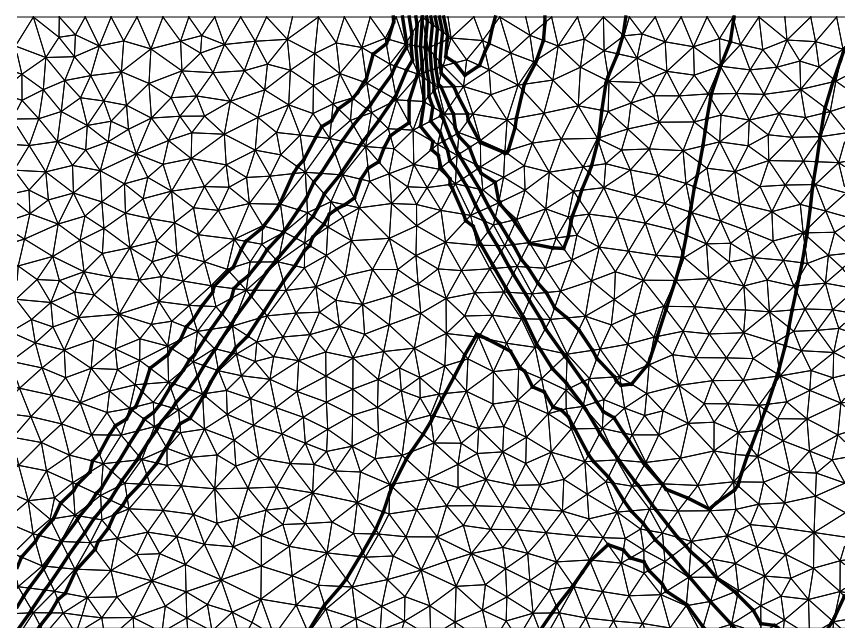

Figure 3. circular-arc bump channel: zoom of the Mach number contours $(\Delta M=$ $0.05)$ and of the grid in the $\lambda$-shock region

The discretized governing equations are solved by means of a four-stage RungeKutta scheme, coupled with an Implicit Residual Smoothing procedure: (Catalano et 
al., 2005b) fully describes the technique here employed to define the smoothing lines on cell-vertex unstructured grids.

A standard V-cycle Full MultiGrid (FMG) (Brandt, 1977) has been also implemented both to accelerate convergence to steady state and to compute the MAFD sensitivities. Finer grids are created during the nested iteration by means of a global uniform refinement.

\section{Blade parameterization}

A good parameterization of the blade profile should allow to explore a wide range of complex and innovative geometries, without slowing down the convergence of the optimization process. To achieve the first of the above requirements, a sufficient number of design parameters is needed. The most obvious choice consists of keeping the mesh points on the blade surface as control points; however, since a perturbation of a design parameter would produce only a local perturbation of the flow solution (thus deteriorating the convergence of the optimization problem), a smoothing of the blade profile is required (Mohammadi et al., 2001), which restricts the flexibility of the representation. A widely employed parameterization consists of combining a number of existing profiles (shape functions), using the corresponding weights as design parameters, see (Dadone et al., 2000a; Kuruvila et al., 1995; Jameson, 1988). An other very common approach is based on the use of interpolation functions, and, in particular, of Spline curves or Bézier-Bernstein polynomials, using some points of the blade or the control points as design parameters, respectively (Farin, 1993).

All cited parameterizations do not use an orthogonal basis as vector of design parameters, which would be the best choice for the optimal convergence of the design problem (Kuruvila et al., 1995) since it avoids to work with a set of functions weakly independent. In this paper, a new method is proposed for the definition of a set of orthogonal shape functions $f_{k}, k=1, \ldots, n_{p}$; orthogonality is, here, defined with respect to the following inner product:

$$
\left(f_{i}, f_{j}\right)=\oint f_{i} f_{j} \mathrm{~d} x
$$

and $n_{p}$ is great as needed for a good representation of complex and innovative aerodynamic shapes $f$. In particular, the method is applied to the design of $2 \mathrm{D}$ turbine blades with wedged trailing edge. With minor modifications, it can be easily applied to the parameterization of blades with rounded trailing edge and of other aerodynamic components, such as compressor blades and airfoils.

A set of airfoil-like profiles $p_{\ell}, \ell=1, \ldots, n_{p}$, is generated by using the BézierBernstein polynomials

$$
\boldsymbol{b}^{n}(q)=\sum_{j=0}^{n} \boldsymbol{b}_{j} \frac{n !}{j !(n-j) !} q^{j}(1-q)^{n-j}, \quad q \in[0,1]
$$


where $\boldsymbol{b}_{j}, j=0, \ldots, n$ contains the coordinates of the $j^{\text {th }}$ Bézier-Bernstein control point. The first $\left(\boldsymbol{b}_{0}\right)$ and the last $\left(\boldsymbol{b}_{n}\right)$ control points coincide, so as to obtain a wedged trailing edge. The Bézier-Bernstein polynomials are here scaled so as to fix the leading edge in $(0,0)$ and the trailing edge in $(1,0)$. Thus, the number of independent profiles, $p_{\ell}$, reduces to $n_{p}=n-2$.

The first curve, $p_{1}$, is obtained by fixing the $(n+1)$ Bézier-Bernstein control points $\boldsymbol{b}_{j}$ so as to draw a reasonable aerodynamic profile. Figure 4 shows the location of the (thirteen) control points employed and the first curve obtained after scaling.

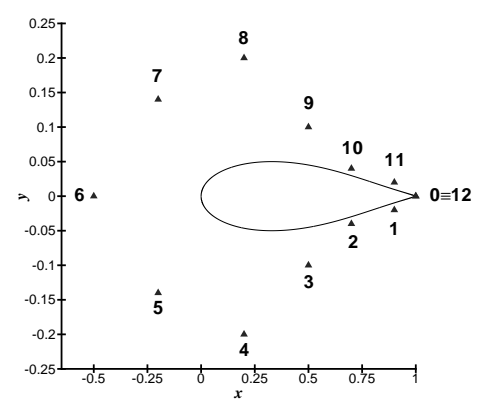

Figure 4. Control points and corresponding Bézier-Bernstein polynomial obtained

Additional $\left(n_{p}-1\right)$ aerodynamic profiles $p_{\ell}$ can be generated by shifting the control points along $y, \boldsymbol{b}_{0}$ and $\boldsymbol{b}_{n}$ remaining unchanged. Then, the set of orthogonal shape functions $f_{\ell}, \ell=1, \ldots, n_{p}$, is finally obtained as follows: the first curve remains unchanged, namely $f_{1}=p_{1}$; the other curves $f_{\ell}, \ell=2, \ldots, n_{p}$, are obtained from the curve $p_{\ell}$ and from the orthogonal shape functions $f_{j}, 1 \leq j<\ell$, previously computed, according to the Gram-Schmidt orthogonalization process:

$$
f_{\ell}=p_{\ell}-\sum_{j=1}^{\ell-1} \frac{\oint p_{\ell} f_{j} \mathrm{~d} x}{\oint f_{j}^{2} \mathrm{~d} x} f_{j}
$$

Finally, the shape functions $f_{\ell}, \ell=2, \ldots, n_{p}$ are empirically scaled so that the maximum of $f_{\ell}$ is equal to the maximum of $f_{1}$.

All computations proposed in this paper employ the six orthogonal profiles shown in Figures 5 and 6.

When considering an airfoil or an axial compressor blade, the orthogonal profiles can be simply combined, using the design variables as weights. For a turbine blade, an alternative procedure is suggested: 


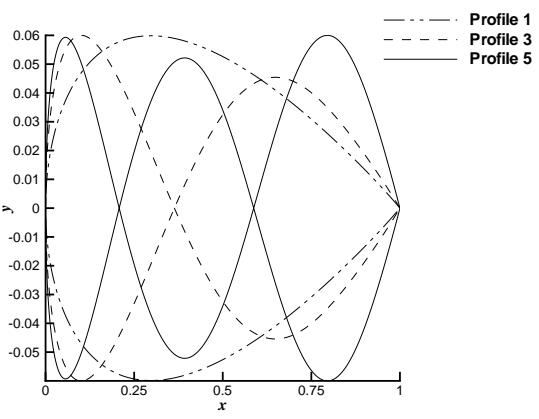

Figure 5. Symmetrical orthogonal shape functions

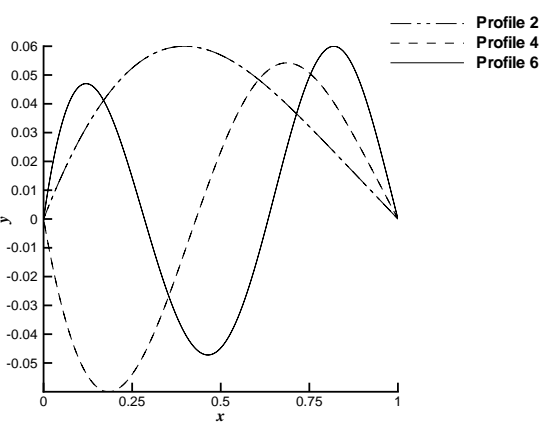

Figure 6. Antisymmetric orthogonal shape functions

1) combine the antisymmetric profiles to define the camberline at each point $i$ :

$$
c^{(i)}=\sum_{\substack{\ell=1 \\ \ell \text { even }}}^{N_{\xi}} \xi_{\ell} f_{\ell}^{(i)} ;
$$

2) rotate the resulting camberline so as to impose the prescribed exit angle; derive a new curve $\mathbf{c}\left(x_{c}^{(i)}, y_{c}^{(i)}\right)$;

3) use the symmetrical profiles to define the blade thickness at each point of the camberline: 


$$
t^{(i)}=\sum_{\substack{\ell=1 \\ \ell \text { odd }}}^{N_{\xi}} \xi_{\ell} f_{\ell}^{(i)}
$$

4) obtain the blade profile by adding the thickness $t^{(i)}$ in the direction normal to each point $i$ of the camberline:

$$
\begin{aligned}
& x^{(i)}=x_{c}^{(i)}+t^{(i)} \cdot n_{x}^{(i)} \\
& y^{(i)}=y_{c}^{(i)}+t^{(i)} \cdot n_{y}^{(i)} .
\end{aligned}
$$

\section{Multigrid-aided finite-difference sensitivities}

The MAFD procedure proposed in this paper aims at reducing the computational work required by the flow computation on perturbed geometries, while maintaining the advantages cited in the Introduction.

According to the so-called dual viewpoint of the multigrid (MG) technique, a constant term is computed at each MG cycle and added to the right-hand side on the coarser grid level. This term can be written as:

$$
\mathcal{R}_{h}\left(\mathcal{R}_{U} \tilde{U}_{h}^{\ell}\right)-\mathcal{R}_{d} \mathcal{R}_{h}\left(\tilde{U}_{h}^{\ell}\right)
$$

where $\mathcal{R}_{U}$ and $\mathcal{R}_{d}$ are suitable restriction operators for the solution and for the defect from the fine level $h$ to the coarse level $H$ and $\mathcal{R}_{h}$ is the flow residual. As known, this term represents an approximate value of the relative local truncation error (RLTE) between the finer grid and the coarser one (Brandt, 1977). Moreover, the method works accordingly to three following important remarks: $i$ ) the multigrid strategy solves the flow equations on coarser grid levels with the same accuracy of the finer level, thanks to the addition of the RLTE term. Moreover, $i i$ ) a correct choice of the design parameters should give a smooth perturbation of the blade profile, that can be seen effectively on a coarser level. Finally, iii) the approximate RLTE, which mainly represents the difference of accuracy between two nested grid levels, is not affected by a small, smooth perturbation of one design parameter.

On the basis of these three considerations, the proposed method allows to compute the difference between the flow solutions of perturbed geometries using a coarser grid level and a value of the RLTE computed only once, using the unperturbed geometry. The entire procedure can be summarized as follows:

1) store the flow solution for the current geometry computed on the finest mesh;

2) compute and store the RLTE between the finest and the coarsest grid solutions for the current configuration, using the same subroutines already written for the MG cycle; set $\ell=1$; 
3) assign small positive and negative perturbations to the $\ell^{\text {th }}$ design parameter;

4) restrict the current fine-mesh flow solution, previously stored, onto the coarsest grid level, without intermediate solver applications;

5) approximately compute (with the same level of convergence) the flow solutions for the perturbed geometries on the coarsest level employed, adding the stored RLTE to the right-hand-side of the flow equations, as done in the MG technique;

6) compute and prolongate (to the finest grid level) the solution corrections;

7) update the flow solution on the finest mesh and compute the objective function;

8 ) in case of discontinuities, smooth the objective function, using a simple highfrequency filter;

9) evaluate the sensitivity derivative as:

$$
D_{\xi_{\ell}} I=\frac{I\left(\xi_{\ell}+\Delta \xi_{\ell}\right)-I\left(\xi_{\ell}-\Delta \xi_{\ell}\right)}{2 \Delta \xi_{\ell}} \approx \frac{\partial I}{\partial \xi_{\ell}}
$$

10) if $\ell<N_{\xi}$, increase $\ell$ of one and go to step 3 .

Centered finite differences have been preferred with respect to one-side differences for robustness, rather than for accuracy. It is noteworthy that almost identical performances are obtained with a step-size $\Delta \xi$ ranging from $10^{-3}$ to $10^{-5}$, provided that the convergence level $\left(\log _{10}\right.$ of the flow residual) of the coarse-grid solution for the perturbed shapes, $R_{p s}$, is related to the current convergence level of the finest grid solution, $R_{f g s}$, by the following empirical relation:

$$
R_{p s}=R_{f g s}-1+0.5\left(3+\log _{10} \Delta \xi\right)
$$

It is also noteworthy that the coarse-grid evaluation of the perturbed flow fields is very efficient, while preserving the fine-grid accuracy: the coarser grid levels have a much lower number of cells and allow the use of a higher time step (the time step is at least doubled at each coarsening). The cost to compute the approximate derivatives still depends on the number of design parameters, $N_{\xi}$, but has been drastically reduced. Moreover, the entire procedure can be easily parallelized: in such a case, the required computational work would become additionally reduced and become possibly independent of $N_{\xi}$ (if $N_{\xi}$ is smaller than the number of available processors).

\section{Progressive optimization}

The simultaneous convergence of the design process and of the flow analysis, also including the global refinement from a coarse to a sufficiently fine mesh, is the basis of the progressive optimization strategy proposed in (Dadone et al., 2000b) and here employed: less accurate sensitivity derivatives (i.e. with partially converged flow solutions computed on coarser levels) are used when the geometry is far from the optimal one; then, the convergence level of the flow solution and the number of mesh points are increased while approaching the optimum. Starting the optimization on coarser grids 
and using partially converged flow solutions drastically reduce the computational cost of the entire optimization procedure, without affecting its robustness and capability of finding the optimum, as demonstrated by the large number of applications proposed so far. This approach is adapted to the MAFD computation of sensitivities here proposed, furnishing the following MAFD progressive optimization algorithm (see also (Catalano et al., 2003a)):

1) start with an initial set of design variables;

2) start the flow computations on a coarse grid;

3) advance the flow solver for several iterations;

4) compute the objective function gradient, $\nabla I$, by means of the MAFD approach;

5) update the design variables according to the relation:

$$
\xi_{\ell}^{l+1}=\xi_{\ell}^{l}-a_{\ell} D_{\xi_{\ell}} I
$$

where $a_{\ell}$ are positive parameters;

6) repeat steps 3 to 5 until the gradient of the objective function is sufficiently decreased (from one to two orders of magnitude);

7) refine the mesh by doubling the number of intervals in each direction and interpolate the computed flow solution to the finer grid;

8) repeat steps 3 to 5 until the gradient of the objective function has decreased half to one order of magnitude more;

9) repeat steps 7 and 8 until the finest grid is reached;

10) repeat steps 3 to 5 until the gradient of the objective function has sufficiently decreased.

The coefficient $a_{\ell}$ in eq. (10) is evaluated as $a_{\ell}=b \cdot c_{\ell}$. The coefficient $b$ represents the minimum value of $a_{\ell}$ and is given by:

$$
b=\frac{\Delta \xi_{c}}{\kappa\left|\nabla_{\ell} I\right|_{\max }^{0}},
$$

where $\Delta \xi_{c}$ is a typical (order of magnitude) change of the design parameters, $\kappa$ is a constant ranging from 40 to 100 and depending on the test case, and $\left|\nabla_{\ell} I\right|_{\max }^{0}$ is the largest absolute value assumed by the sensitivity derivatives of the objective function after the first global step through steps 1 to 5 . At the beginning of the computations, $c_{\ell}$ is set to 1 , then it is increased by $50 \%$ if the corresponding sensitivity derivative of the objective function maintains its sign, while it is decreased by $50 \%$ if the sign changes. This approach allows large changes for the design variables whose sensitivity derivatives maintain the same sign, while it assigns small changes to design variables whose sensitivity derivatives are changing their sign. This technique is more efficient than the method of steepest descent, corresponding to $c_{\ell}$ equal to one, due to the approximate gradient evaluation. 


\section{Validation of the solver for turbulent computations}

The well-know transonic flow about the RAE2822 airfoil reported in (Cook et al., 1979) as Case 9, has been considered to test the flow solver employed for turbulent computations (see (Catalano et al., 2003b)). The undisturbed Mach number is $M_{\infty}=0.73$, the angle of attack is $2.8^{\circ}$ and the Reynolds number is $R e=6.5 \times 10^{6}$. The grid employed is obtained by subdividing a C-mesh with $256 \times 64$ cells ( 160 points are on the surface). A constant stretching is imposed in direction normal to the surface, the resulting $y^{+}$being always less than 1 . Figure 7 shows the Mach number contours computed by the present method, using the circumcenters to generate the dual mesh. Figure 8 provides the corresponding distribution of the pressure coefficient. The comparison with the experimental results of (Cook et al., 1979) is rather good, except in the shock region, as also found by other authors. Finally, Figure 9 reports the distribution of the skin friction coefficient (non-dimensionalized with respect to the free-stream conditions), which also in this case is in good agreement with the experimental data (computed from those provided in (Cook et al., 1979) with a different definition), except beyond the shock. Velocity profiles at different axial stations are provided in (Catalano et al., 2003b).

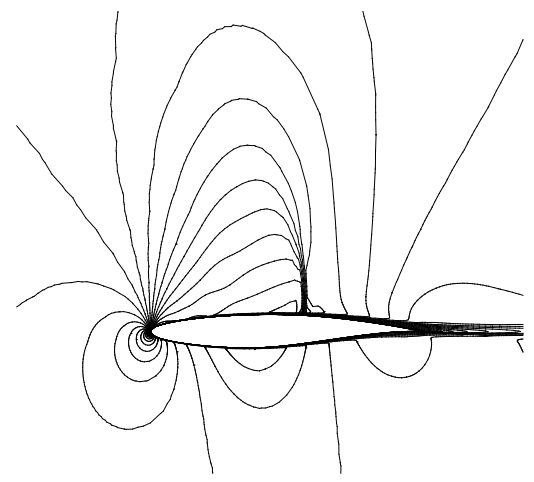

Figure 7. RAE-2822 airfoil: Mach number contours $(\Delta M=0.05)$

\section{Results}

The inverse design of a 2D turbine blade, in both inviscid and turbulent transonic flow conditions, has been approached in this paper to validate the proposed strategy, namely to check its capability to reach the optimum and its efficiency: the target shape, defined by known values of the design parameters $\xi_{j}$, is used to compute a 
target pressure distribution, $\hat{p}$, that must be matched by the pressure distribution $p$ computed on the trial shape: accordingly, the objective function is defined as

$$
I(\boldsymbol{\xi})=\frac{1}{2 S} \int_{S}[p-\hat{p}]^{2} \mathrm{~d} S
$$

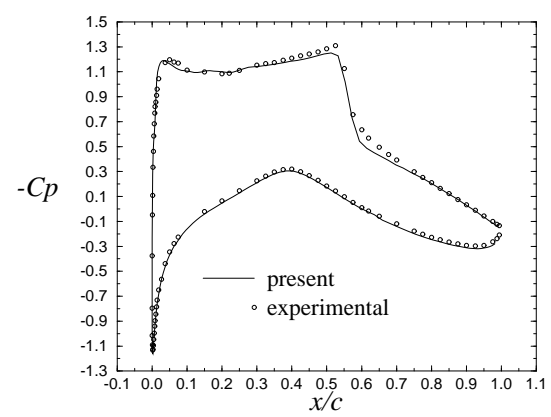

Figure 8. RAE-2822 airfoil: pressure coefficient

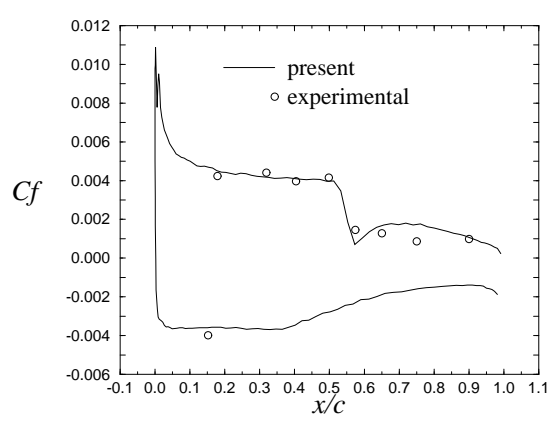

Figure 9. RAE-2822 airfoil: skin friction coefficient

All applications have been obtained with the same code and with the addition of a simple high-frequency filtering (smoothing) of the target and of the computed pressure distributions, when evaluating the sensitivities, since a sharp shock is captured by the flow solver on the suction side of the blade. The known target blade has been preliminary defined by combining the six orthogonal (three symmetric and three antisymmetric) base functions provided in Figure 5 and 6, with weights (or design parameters) $\xi_{1}=2.2, \xi_{2}=3.5, \xi_{3}=-0.4, \xi_{4}=-0.1, \xi_{5}=-0.1$ and $\xi_{6}=0.1$, see the solid line plotted in Figure 10. In all cases, the initial profile, plotted as a dashed line in Figure 10, is defined by the design parameters $\xi_{1}=1.5, \xi_{2}=5.0, \xi_{3}=0.0$, $\xi_{4}=0.0, \xi_{5}=0.0$ and $\xi_{6}=0.0$. Both the $\mathrm{MG}$ and the progressive optimization 
employ three grid levels, the finest mesh being composed of 9351 nodes and 18432 triangles (97 nodes on the blade).

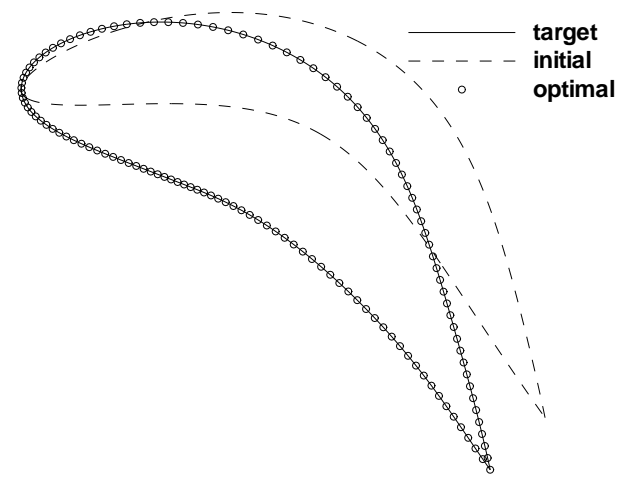

Figure 10. Target, initial and optimal profiles

Transonic inviscid flow conditions have been considered for the first test-case: incidence angle $\alpha=0^{\circ}$, outlet isoentropic Mach number $M_{2, \text { is }}=0.9$. The Mach number contours and the target pressure distribution for this test-case are shown in Figures 11 and 12, respectively, a sharp shock being located on the rear part of the suction side.

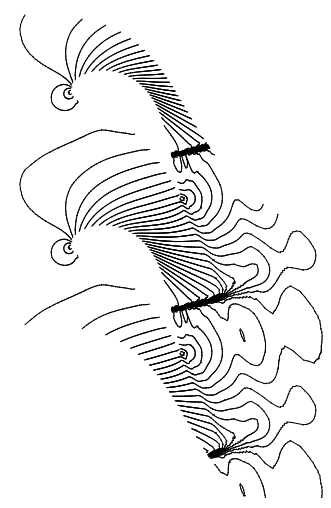

Figure 11. Mach number contours (inviscid flow conditions) $\Delta M=0.05$ 


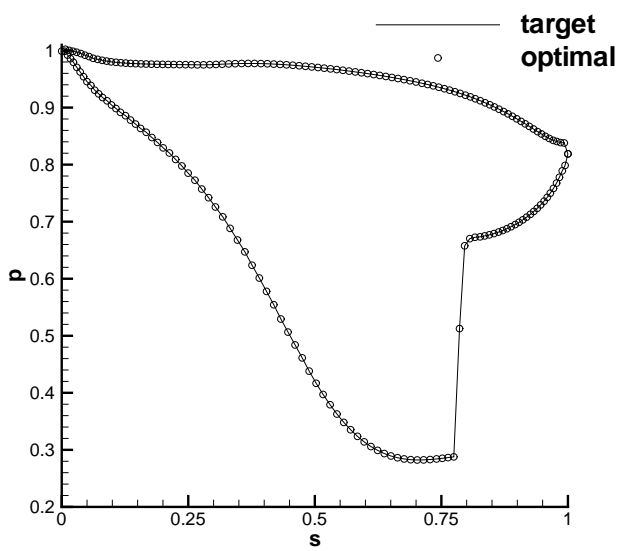

Figure 12. Target pressure distribution (inviscid flow conditions)

Figure 13 proposes the convergence histories of the flow residual, of the objective function and of the magnitude of the objective function gradient. One work unit is defined as one (converged) MG computation of the target flow.

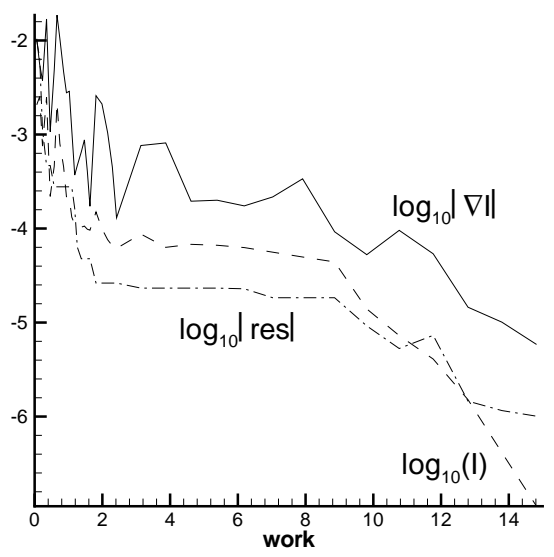

Figure 13. Convergence histories (inviscid flow conditions)

The optimization procedure is firstly applied on the coarsest level, which cannot take advantage of the MAFD procedure. Two grid refinements have been set both for $\log _{10}|\nabla I| \leq-3.5$. The whole optimization process is stopped when $\log _{10}|\nabla I| \leq$ -5.0 at the finest level, which is even excessive for engineering applications. The 
first grid refinement is performed after 1.6 work units. At the second grid level, the MAFD technique employs the coarser mesh to compute the sensitivity derivatives and the second refinement is located at work $\approx 2.4$. Figures 13 indicates that the work required to obtain the more than satisfactory convergence level of -5.0, on the finest mesh, is about 15. The optimal blade (symbols) is perfectly superposed to the target configuration (solid line), see Figure 10. As well, the optimal pressure distribution accurately matches the target pressure distribution, see Figure 12.

The MAFD progressive optimization has been finally tested under turbulent flow conditions, using the same target and initial profiles: $R e=10^{6}$, outlet isoentropic Mach number $M_{2, i s}=0.75$, incidence angle $\alpha=0^{\circ}$, inlet turbulence intensity $T u=$ $4 \%$. Figure 14 provides the corresponding Mach number contours $\left(y^{+}\right.$always lower than one). The target pressure distribution is shown in Figure 15.

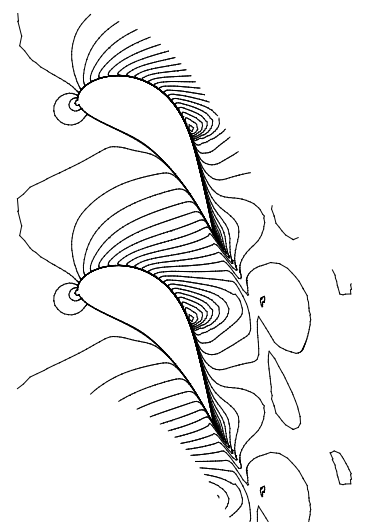

Figure 14. Mach number contours (turbulent flow conditions) $\Delta M=0.05$

Figure 16 provides the convergence histories of the optimization. The two grid refinements are performed after 3 and 5 work units, respectively. Figure 16 indicates that the work required to obtain the fully satisfactory convergence level of -5.0 , on the finest mesh, is about 20. Also in this case, the optimal pressure distribution matches the target one, see Figure 15.

\section{Conclusions}

A very efficient and robust progressive-optimization procedure using MultigridAided Finite-Differences for the computation of the sensitivity derivatives has been proposed and combined with an upwind finite-volume method for the Euler and the 
Navier-Stokes equations on cell-vertex unstructured triangular grids. The entire approach is cheap, flexible and easy-to-program; it also turns out to be robust and highly efficient, the converged design of a 2D turbine blade, under inviscid and turbulent transonic flow conditions, being obtained in a computational time equal to that required by 15 to 20 multigrid flow analyses on the finest grid.

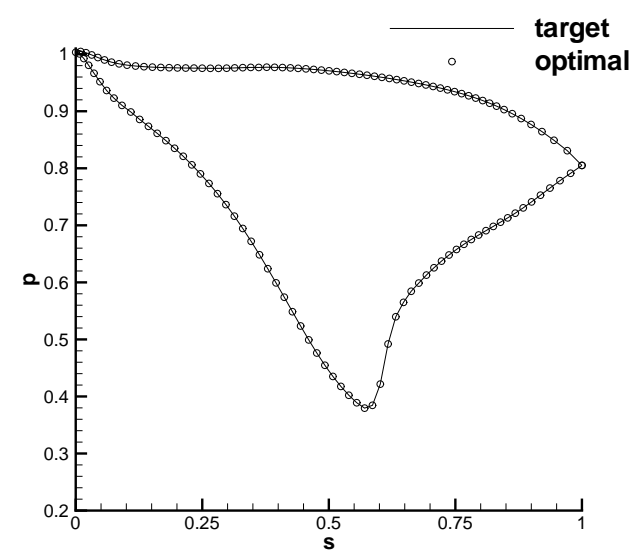

Figure 15. Target pressure distribution (turbulent flow conditions)

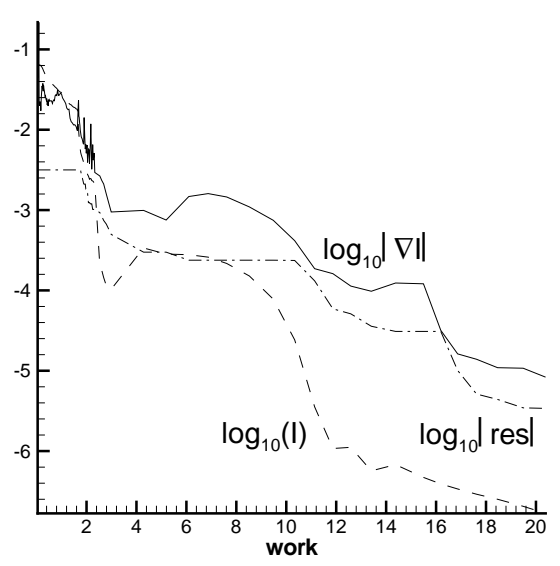

Figure 16. Convergence histories (turbulent flow conditions) 


\section{References}

Beux F., Dervieux A., “ A hierarchical approach for shape optimization”, Eng. Comput., vol. 11, n 6, p. 25-48, 1994.

Brandt A., "Multi-level adaptive solutions to boundary-value problems”, Math. Comp., vol. 31, p. 333-390, 1977.

Catalano L. A., " A new reconstruction scheme for the computation of inviscid compressible flows on 3D unstructured grids", International Journal for Numerical Methods in Fluids, vol. 40, n 1-2, p. 273-279, 2002.

Catalano L. A., Dadone A., Daloiso V. S. E., “Progressive optimization on unstructured grids using multigrid-aided finite-difference sensitivities", International Journal For Numerical Methods In Fluids, vol. 47, n 10-11, p. 1383-1391, 2005a.

Catalano L. A., Dadone A., Daloiso V. S. E., Mele G., Progressive optimization using orthogonal shape functions and efficient finite-difference sensitivities, AIAA Paper 2003-3961, 2003a.

Catalano L. A., Daloiso V. S. E., Accurate computation of $2 D$ turbulent compressible flows on unstructured grids, AIAA Paper 2003, 2003b.

Catalano L. A., Daloiso V. S. E., “ Upwinding and Implicit Residual Smoothing on cell-vertex unstructured grids”, International Journal For Numerical Methods In Fluids, vol. 47, n 8-9, p. 895-902, 2005 b.

Cook P. H., McDonald M. A., Firmin M. C. P., “ Aerofoil RAE 2822 - Pressure Distributions, and Boundary Layer and Wake Measurements”, AGARD AR-138, p. A6.1-A6.77, 1979.

Dadone A., Grossman B., " Fast convergence of viscous airfoil design problems”, AIAA paper 2000-2547, 2000a.

Dadone A., Grossman B., “ Progressive Optimization of Inverse Fluid Dynamic Design Problems", Computers and Fluids, vol. 29, p. 1-32, 2000 b.

Farin G., Curves and surfaces for computer aided geometric design. A practical guide, Third edition. Academic Press, Inc., 1993.

Held C., Dervieux A., “ One-shot airfoil optimisation without adjoint”, Comput. Fluids, vol. $31, n^{\circ} 8$, p. 1015-1049, 2002.

Jameson A., “ Aerodynamic design via control theory”, SIAM J. Sci. Comput., vol. 3, p. 233 260,1988

Kuruvila G., Ta'asan S., Salas M. D., Airfoil desing and optimization by the one-shot method., AIAA Paper 95-0478, 1995.

Mohammadi B., " A new optimal shape design procedure for inviscid and viscous turbulent flows", Int. J. Numer. Meth. Fluids, vol. 25, p. 183-203, 1997.

Mohammadi B., Pironneau O., Applied shape optimization for fluids., Oxford Science Publications, Clarendon Press., 2001.

Roe P. L., "Characteristic based schemes for the Euler equations", Ann. Rev. Fluid Mech, vol. 18, p. 337-365, 1986.

Wilcox D. C., " Reassessment of the scale-determining equation for advanced turbulence models”, AIAA Journal, vol. 26, n 11, p. 1299-1310, 1988. 
